\title{
Clinical and Polysomnographic Characteristics of Patients with Extreme Obstructive Sleep Apnea, AHI > 100: A Case-Control Study
}

\author{
Forogh Soltaninejad ${ }^{1}$, Negarsadat Neshat ${ }^{2}$, Mehrzad Salmasi ${ }^{3,{ }^{*}}$ and Babak Amra ${ }^{4}$ \\ ${ }^{1}$ Respiratory Research Center, Department of Internal Medicine, School of Medicine, Isfahan University of Medical Sciences, Isfahan, Iran \\ ${ }^{2}$ Faculty of Medicine, Isfahan University of Medical sciences, Isfahan, Iran \\ ${ }^{3}$ Department of Internal Medicine, School of Medicine, Isfahan University of Medical Sciences, Isfahan, Iran \\ ${ }^{4}$ Bamdad Respiratory Research Center, Isfahan University of Medical Sciences, Isfahan, Iran \\ "Corresponding author: Department of Internal Medicine, School of Medicine, Isfahan University of Medical Sciences, Isfahan, Iran. Email: mehrzad_salmasi@yahoo.com
}

Received 2020 September 20; Revised 2020 November 21; Accepted 2020 November 28.

\begin{abstract}
Background: Severe obstructive sleep apnea (OSA), defined by apnea-hypopnea index (AHI) as more than 30 events per hour, was previously related to more comorbidity. However, limited studies separated the patients with AHI $>100$ from those with a less severe manifestation of the disease.

objectives: The current study aimed at describing the characteristics of this subgroup and comparing them with less severe conditions.

Methods: A retrospective analysis was conducted on 114 patients with OSA. Nocturnal polysomnography was used to diagnose severe OSA. Patients were categorized into two groups: (1) $60<$ AHI $<100$ (very severe OSA), (2) AHI $\geq 100$ (extreme OSA). Demographic, medical history, and polysomnographic variables were evaluated and compared between the two groups.

Results: Extreme OSA was diagnosed in 19 patients, the mean body mass index (BMI) was significantly higher in this group ( $39.26 \pm$ 5.93 vs. $\left.35.68 \pm 6.45 \mathrm{~kg} / \mathrm{m}^{2}, \mathrm{P}=0.025\right)$. They also had lower minimal $\mathrm{O}_{2}$ saturation $(65.68 \pm 10.16 \mathrm{vs} .74 .10 \pm 8.74, \mathrm{P}=0.003)$ and more time with $<90 \% \mathrm{O}_{2}$ saturation $(\mathrm{T}<90 \%)(81.78 \pm 22.57 \mathrm{vs.} 58.87 \pm 33.14, \mathrm{P}=0.01)$. OHS prevalence was significantly higher in the group with extreme OSA $(\mathrm{P}=0.04)$. The most frequent comorbidity was hypertension, with an incidence of $60.5 \%$, for the extreme group, although there was no significant difference between the two groups in terms of clinical associations.

Conclusions: The current study results suggested that greater BMI and lower minimal $\mathrm{O}_{2}$ saturation, as well as increased $\mathrm{T}<90 \%$, were associated with extreme OSA, although no differences were observed in the associated diseases between the compared groups.
\end{abstract}

Keywords: Obstructive Sleep Apnea, Apnea-hypopnea Index, Polysomnography

\section{Background}

Obstructive sleep apnea (OSA) is a common breathing disorder among individuals, and its prevalence ranges from $0.1 \%$ to $13 \%$ (1). The main risk factors of the disease are older age, male gender, and obesity (2). OSA is defined by repeated episodes of airway collapses during sleep time, which can be complete (apnea) or incomplete (hypopnea) (3). Apnea hypopnea index (AHI) is a measure to determine the severity of the disease. AHI ranges of $5-14,15-29$, and $\geq 30$ refer to mild, moderate, and severe disease conditions (4), respectively. The burden of the disease is high due to both healthcare costs related to OSA alone and its contribution as an independent risk factor for metabolic, cardiovascular, and psychiatric disorders (5), such as arterial hypertension, heart failure, and stroke, as well as several metabolic dysfunctions such as DM, glucose intolerance, and insulin resistance (6-8).
It is well known that the morbidity and mortality rate is higher in patients with severe OSA compared with those with mild to moderate disease, especially in patients with underlying diseases, such as arterial hypertension, coronary artery disease, and stroke $(7,9)$. There is convincing data to support early treatment effects on the reduction of symptoms and cardiovascular risks in such patients (10). Hence, early diagnosis and management is particularly important in severe cases. However, severe OSA includes a wide range of patients with $\mathrm{AHI} \geq 30$, and limited studies investigated clinical and polysomnographic characteristics of different subsets of this group. Jurcevic D. et al., compared patients with AHI $\geq 60$ to those with less severe disease and suggested that patients with AHI $\geq 60$ are more likely older obese males with lower total sleep time with $<90 \% \mathrm{O}_{2}$ saturation $(\mathrm{T}<90 \%)$. They also demonstrated that this group of patients has an overall higher 
mortality rate (9). In addition, de Castro et al., also claimed that arterial HTN, neck circumstance, age, and over $10 \%$ of $\mathrm{T}<90 \%$ are associated with $\mathrm{AHI} \geq 100$ (11). Yet, no comparisons with other associated diseases and polysomnographic characteristics of patients with AHI $\geq 100$ are reported. Therefore, it is beneficial to define the prognostic variables for this subset of severe OSA.

\section{Objectives}

The current retrospective study aimed at investigating whether associated diseases and/or risk factors are higher in patients with $\mathrm{AHI} \geq 100$ than those with $60>\mathrm{AHI}>100$ was performed.

\section{Methods}

\subsection{Study Design and Participants}

The current retrospective study was conducted on adult patients referring to Bamdad Sleep Clinic in Isfahan, Iran, from 2018 to 2020 for the evaluation of suspected OSA. Patients undergoing eight hours of split night polysomnography (PSG) and diagnosed with very severe sleep apnea (AHI $\geq 60$ per hour) were selected. Patients with other medical neurodegenerative or psychiatric problems that might affect their sleep quality, such as congestive heart failure, myasthenia gravis, or major depressive disorder, were excluded. The AASM (American Academy of Sleep Medicine) Sleep Scoring Manual version 2.6 was used to score the subjects. Two OSA subgroups were defined as very severe sleep apnea (very severe OSA) $(60 \leq \mathrm{AHI}<100)$ and extremely severe sleep apnea (extreme OSA) (AHI $\geq$ 100).

\subsection{Procedure and Variable Assessments}

In the current study, standard PSG equipment(Embla®) N7000 Recording System PSG) for OSA diagnosis was utilized by which parameters, such as electroencephalography, electro-oculography, electromyography, electrocardiography, oxygen saturation, respiratory effort, oronasal airflow and snoring sounds, and body position, were recorded.

The reported parameters included AHI, which is the average number of apnea and hypopnea episodes per hour; minimal $\mathrm{O}_{2}$ saturation, which is the lowest $\mathrm{SpO}_{2}$ during sleep; $\mathrm{T}<90$, which is the total time of sleep with less than $90 \% \mathrm{O}_{2}$ saturation, and central apnea percentage, which is the ratio of central apneas to total apneas in one hour. Sleep scoring criteria for the staging system were determined according to AASM (12).
Clinical characteristics were obtained from the medical records of patients. In some cases, there was no documented medical history, and the data were obtained by self-reporting of drugs taken for hypertension, hypothyroidism, hyperlipidemia, or diabetes.

\subsection{Statistical Analysis}

Statistical analysis was performed using SPSS version 18 software. Numerical measures were expressed as mean \pm standard deviation(SD). Clinical and polysomnographic findings were compared between the two groups using $t$ test for quantitative and chi-square test for categorical variables. A P-value of $<0.05$ was considered the level of significance.

The current study was approved by the Ethics Committee of the Institutional Board Review of Isfahan University of Medical Sciences, Isfahan, Iran (ethical code; IR.MUI.MED.REC.1398.625).

\section{Results}

Out of the total 114 patients with OSA included in the study, 19 had $\mathrm{AHI} \geq 100$ and $9560 \leq \mathrm{AHI}<100$, with the mean AHI of 113.95 in extreme OSA and 75.66 in severe OSA groups.

The mean age of participants was 60.36 years, and the mean BMI $36.27 \mathrm{~kg} / \mathrm{m}^{2}$. The baseline characteristics were compared according to AHI among 114 patients, and it was observed that those with extreme OSA were more obese than patients with severe OSA (39.26 \pm 5.93 vs. $35.68 \pm 6.45$ $\left.\mathrm{kg} / \mathrm{m}^{2}, \mathrm{P}=0.025\right)$. Obesity hypoventilation syndrome(OHS) was significantly higher in the extreme OSA group (48\% vs. $84 \%, \mathrm{P}=0.04$ ) (Table 1). Moreover, compared to the severe OSA group, the extreme OSA group had a lower minimal $\mathrm{O}_{2}$ saturation ( $65.68 \pm 10.16$ vs. $74.10 \pm 8.74, \mathrm{P}=0.003)$ and a higher $\mathrm{T}<90$ to total sleep time ( $81.78 \pm 22.57 \mathrm{vs.} 58.87 \pm$ 33.14, $\mathrm{P}=0.01$ ) (Table 2).

Among the 114 study subjects, 69 (60.5\%) had hypertension, 35 (30.7\%) diabetes mellitus (DM), 41 (36.0\%) hyperlipidemia, 10 (8.8\%) a history of PCI, and 10 (8.8\%) hypothyroidism, although no significant differences were observed regarding these clinical parameters (Table 1 ).

Of the 19 patients in the extreme OSA group, 12 (63.2\%) responded to Bilevel positive airway pressure (PAP) and seven $(36.8 \%)$ to continuous positive airway pressure therapies. Compared to patients in the very severe group, those with AHI $\geq 100$ had a better response to Bilevel PAP, although the difference between the two groups was insignificant (63.2\% vs. $40 \% \mathrm{P}=0.063)$ (Table 2 ). 
Soltaninejad $\mathrm{F}$ et al.

\begin{tabular}{|c|c|c|c|}
\hline & Very Severe OSA, N= 95 & Extreme OSA, $\mathrm{N}=19$ & P-Value \\
\hline Age $(y)$ & $60.68 \pm 11.10$ & $58.74 \pm 13.20$ & 0.528 \\
\hline Gender (M/F), No. (\%) & $53(55.8) / 42(44.2)$ & $11(57.9) / 8(42.1)$ & 0.866 \\
\hline Body mass index, $\mathrm{kg} / \mathrm{m}^{2}$ & $35.68 \pm 6.45$ & $39.26 \pm 5.93$ & $0.025^{\mathrm{a}}$ \\
\hline Hypertension, No. (\%) & $55(57.9)$ & $14(73.7)$ & 0.199 \\
\hline Diabetes mellitus, No. (\%) & $27(28.4)$ & $8(42.1)$ & 0.238 \\
\hline Hyperlipidemia, No. (\%) & $32(33.7)$ & $9(47.4)$ & 0.257 \\
\hline PCI, No. (\%) & $7(7.4)$ & $3(15.8)$ & 0.236 \\
\hline Hypothyroidism, No. (\%) & $9(9.5)$ & $1(5.3)$ & 0.554 \\
\hline $\mathrm{OHS}^{\mathbf{b}}$ & $46(48 \%)$ & $16(84 \%)$ & $0.04^{\mathrm{a}}$ \\
\hline \multicolumn{4}{|c|}{$\begin{array}{l}\text { a significant difference } \\
\text { bobesity hypoventilation syndrome }\end{array}$} \\
\hline & Very Severe OSA $(\mathrm{N}=95)$ & Extreme OSA $(\mathrm{N}=19)$ & P-Value \\
\hline Central apnea index, $(\%)$ & $7.40 \pm 10.31$ & $4.88 \pm 6.17$ & 0.308 \\
\hline AHI & $75.6 \pm 11.1(73.5-77.9)$ & $113.9 \pm 2.8$ (108.1-119.9) & $0.01^{\mathrm{a}}$ \\
\hline Minimal 02 saturation, (\%) & $74.10 \pm 8.74$ & $65.68 \pm 10.16$ & $0.003^{\mathrm{a}}$ \\
\hline $\mathrm{T}<\mathbf{9 0}$ to $\mathrm{TST},(\%)$ & $58.8 \pm 33.14$ & $81.78 \pm 22.57$ & $0.001^{\mathrm{a}}$ \\
\hline CPAP, No. (\%) & $57(60.0)$ & $7(36.8$ & 0.063 \\
\hline BIPAP, No (\%) & $38(40.0)$ & $12(63.2)$ & 0.063 \\
\hline
\end{tabular}

${ }^{\mathrm{a}}$ significant difference

\section{Discussion}

The main goal of the present retrospective study was to determine the clinical and polysomnographic differences between the two subgroups of severe OSA, as well as the baseline characteristics of the patients, such as age, gender, and obesity. Knowledge of different characteristics of patients with AHI > 100 may increase awareness and improve the diagnosis. The present study assessed the differences in clinical and polysomnographic findings between 19 patients with AHI $\geq 100$ and 95 with $60 \leq \mathrm{AHI}<100$, and it was observed that patients with extreme AHI were significantly more obese, had lower minimal $\mathrm{O}_{2}$ saturation during sleep, and relatively more time with $\mathrm{O}_{2}$ saturation $<90 \%$.

Obesity is a major risk factor for OSA presented in 70\% of patients with this disorder, according to previous research $(13,14)$. Lee et al., suggested that obesity might play a pivotal role in the severity of OSA (15).

The current study results were in agreement with those of previous studies, but the specific subgroup of severe apnea, with AHI > 100, was not distinguished in the literature. One of the very few studies in which patients with
AHI $>100$ were distinguished from other subgroups of severe OSA was conducted by de Castro et al. (11). They investigated patients with AHI $>100$ and, in a bivariate analysis, found an intense association between obesity and extreme OSA. However, the association was lost when neck circumstance was adjusted; it was not observed in the current study.

Previous studies also showed that the degree of nocturnal hypoxemia was higher in patients with severe OSA $(11,16)$. This parameter is evaluated by indicators, such as $\mathrm{T}<90 \%$ and minimal $\mathrm{O}_{2}$ saturation. Kulkas et al., demonstrated that the minimal $\mathrm{O}_{2}$ saturation decreases as tending toward severe OSA (17) that was in agreement with the current study findings, and the lowest $\mathrm{SpO}_{2}$ and $\mathrm{T}<$ 90 were significantly different in patients with extreme OSA, which should be considered as a great risk factor for cardiovascular events and apnea-induced hypertension in this subgroup (17-20). Gehring et al., evaluated patients with severe OSA and found that nocturnal BP fluctuations, along with very low $\mathrm{O}_{2}$ saturation, can add to cardiovascular events during the night (17). It is well known that intermittent hypoxia in patients with OSA contributes to the pathogenesis of hypertension by activating the rennin- 
angiotensin system (18). Severity in oxygen deterioration is also related to impaired glucose tolerance in patients with OSA (20). Thus, the mentioned complications indicate the importance of severe nocturnal hypoxia management in patients with extreme OSA.

Despite the fact that patients in the extreme OSA group had a higher response to Bilevel PAP, the difference was not statistically significant, which could be due to the small number of patients in the extreme OSA group.

Higher arterial blood pressure is previously shown in association with AHI >100 compared to moderate (AHI 15 - 29) and severe (AHI 30 - 50) cases (21), but in the current study, hypertension prevalence was not significantly different among the subgroups of severe OSA.

The main limitation of the present study was the small sample size due to a low number of cases referred to the study site and diagnosed by polysomnography with very severe apnea. However, to avoid losing statistical power, the number of controls was increased. Additionally, the clinical characteristics of all the patients could not be evaluated due to the missing documents or lack of selfknowledge of patients.

\subsection{Conclusion}

In the present study, patients with extreme OSA (AHI $>100$ ) were significantly more obese and had different polysomnographic manifestations, such as lower minimal $\mathrm{O}_{2}$ saturation and more time with $<90 \% \mathrm{O}_{2}$ saturation, compared to those with $60<\mathrm{AHI}<100$.

\section{Footnotes}

Authors' Contribution: FS: The conception of the work, editing of the manuscript, data gathering, and analysis of data; NS: Editing of the manuscript, data gathering, and analysis of data; MS:Editing of the manuscript, data gathering, and analysis of data; BA: editing the manuscript, data gathering, and analysis of data. The authors approved all aspects of the work.

Clinical Trial Registration Code: it is not clinical trial Conflict of Interests: The authors declared no conflicts of interest.

Ethical Approval: The study protocol was approved by the Ethics Committee of the Institutional Board Review of Isfahan University of Medical Sciences, Isfahan, Iran (ethical code: IR.MUI.MED.REC.1398.625).

Funding/Support: There was no funding/support for the present study.

Informed Consent: Informed consent was not applicable to the present study.

\section{References}

1. Memon J, Manganaro SN. Obstructive Sleep-disordered Breathing StatPearls. Treasure Island (FL); 2021. eng.

2. Fietze I, Laharnar N, Obst A, Ewert R, Felix SB, Garcia C, et al. Prevalence and association analysis of obstructive sleep apnea with gender and age differences - Results of SHIP-Trend. J Sleep Res. 2019;28(5). e12770. doi: 10.1111/jsr.12770. [PubMed: 30272383].

3. Jordan AS, McSharry DG, Malhotra A. Adult obstructive sleep apnoea. Lancet. 2014;383(9918):736-47. doi: 10.1016/S0140-6736(13)607345. [PubMed: 23910433]. [PubMed Central: PMC3909558].

4. Ruehland WR, Rochford PD, O’Donoghue FJ, Pierce RJ, Singh P, Thornton AT. The new AASM criteria for scoring hypopneas: impact on the apnea hypopnea index. Sleep. 2009;32(2):150-7. doi: 10.1093/sleep/32.2.150. [PubMed: 19238801]. [PubMed Central: PMC2635578].

5. Basoglu OK, Tasbakan MS. Gender differences in clinical and polysomnographic features of obstructive sleep apnea: a clinical study of 2827 patients. Sleep Breath. 2018;22(1):241-9. doi: 10.1007/s11325-017-1482-9. [PubMed: 28197893].

6. Shepard JJ. Hypertension, cardiac arrhythmias, myocardial infarction, and stroke in relation to obstructive sleep apnea. Clin Chest Med. 1992;13(3):437-58. [PubMed: 1521412].

7. Yaggi HK, Concato J, Kernan WN, Lichtman JH, Brass LM, Mohsenin V. Obstructive sleep apnea as a risk factor for stroke and death. $N$ Engl J Med. 2005;353(19):2034-41. doi: 10.1056/NEJMoa043104. [PubMed: 16282178].

8. Peppard PE, Young T, Palta M, Skatrud J. Prospective study of the association between sleep-disordered breathing and hypertension. $N$ Engl J Med. 2000;342(19):1378-84. doi: 10.1056/NEJM200005113421901. [PubMed: 10805822].

9. Jurcevic D, Shaman Z, Krishnan V. A new category: Very severe obstructive sleep apnea has worse outcomes on morbidity and mortality. Chest. 2012;142(4). doi: 10.1378/chest.1389995.

10. Heinzer R, Vat S, Marques-Vidal P, Marti-Soler H, Andries D, Tobback N, et al. Prevalence of sleep-disordered breathing in the general population: the HypnoLaus study. Lancet Respir Med. 2015;3(4):310-8. doi: 10.1016/S2213-2600(15)00043-0. [PubMed: 25682233]. [PubMed Central: PMC4404207].

11. Iber C. The AASM manual for the scoring of sleep and associated events: Rules. Terminology and Technical Specification. 2007.

12. Malhotra A, White DP. Obstructive sleep apnoea. The Lancet. 2002;360(9328):237-45. doi: 10.1016/s0140-6736(02)09464-3.

13. Young T, Palta M, Dempsey J, Skatrud J, Weber S, Badr S. The occurrence of sleep-disordered breathing among middle-aged adults. $N$ Engl J Med. 1993;328(17):1230-5. doi: 10.1056/NEJM199304293281704. [PubMed: 8464434].

14. Lee SA, Lee GH, Chung YS, Kim WS. Clinical, polysomnographic, and CPAP titration features of obstructive sleep apnea: Mixed versus purely obstructive type. J Neurol Sci. 2015;355(1-2):150-4. doi: 10.1016/j.jns.2015.06.005. [PubMed: 26066559].

15. Bojarun A, Vieversyte Z, Jaruseviciene R, Galgauskas S, Asoklis R, Zablockis R. Effect of obstructive sleep apnea on corneal morphological characteristics. Cornea. 2019;38(12):1576-81. doi: 10.1097/ICO.0000000000002069. [PubMed: 31356414].

16. Kulkas A, Muraja-Murro A, Tiihonen P, Mervaala E, Toyras J. Morbidity and mortality risk ratios are elevated in severe supine dominant OSA: a long-term follow-up study. Sleep Breath. 2015;19(2):653-60. doi: 10.1007/s11325-014-1071-0. [PubMed: 25367548].

17. Gehring J, Gesche H, Drewniok G, Kuchler G, Patzak A. Nocturnal blood pressure fluctuations measured by using pulse transit time in patients with severe obstructive sleep apnea syndrome. Sleep Breath. 2018;22(2):337-43. doi: 10.1007/s11325-017-1555-9. [PubMed: 28828627]. 
18. Foster GE, Hanly PJ, Ahmed SB, Beaudin AE, Pialoux V, Poulin MJ. Intermittent hypoxia increases arterial blood pressure in humans through a Renin-Angiotensin system-dependent mechanism. Hypertension. 2010;56(3):369-77. doi: 10.1161/HYPERTENSIONAHA.110.152108. [PubMed: 20625082].

19. Goncalves SC, Martinez D, Gus M, de Abreu-Silva EO, Bertoluci C, Dutra I, et al. Obstructive sleep apnea and resistant hypertension: a case-control study. Chest. 2007;132(6):1858-62. doi: 10.1378/chest.071170. [PubMed: 18079220].
20. Punjabi NM, Sorkin JD, Katzel LI, Goldberg AP, Schwartz AR, Smith PL. Sleep-disordered breathing and insulin resistance in middle-aged and overweight men. Am J Respir Crit Care Med. 2002;165(5):677-82. doi: 10.1164/ajrccm.165.5.2104087. [PubMed: 11874813].

21. Rey de Castro J, Huamani C, Escobar-Cordoba F, Liendo C. Clinical factors associated with extreme sleep apnoea [AHI>100 events per hour] in Peruvian patients: A case-control study-A preliminary report. Sleep Sci. 2015;8(1):31-5. doi: 10.1016/j.slsci.2015.03.002. [PubMed: 26483940]. [PubMed Central: PMC4608882]. 